\title{
Focused Ultrasound for Neuromodulation
}

\author{
David P Darrow ${ }^{1}$ (D) \\ Published online: 28 November 2018 \\ (C) The American Society for Experimental NeuroTherapeutics, Inc. 2018
}

\begin{abstract}
For more than 70 years, the promise of noninvasive neuromodulation using focused ultrasound has been growing while diagnostic ultrasound established itself as a foundation of clinical imaging. Significant technical challenges have been overcome to allow transcranial focused ultrasound to deliver spatially restricted energy into the nervous system at a wide range of intensities. High-intensity focused ultrasound produces reliable permanent lesions within the brain, and low-intensity focused ultrasound has been reported to both excite and inhibit neural activity reversibly. Despite intense interest in this promising new platform for noninvasive, highly focused neuromodulation, the underlying mechanism remains elusive, though recent studies provide further insight. Despite the barriers, the potential of focused ultrasound to deliver a range of permanent and reversible neuromodulation with seamless translation from bench to the bedside warrants unparalleled attention and scientific investment. Focused ultrasound boasts a number of key features such as multimodal compatibility, submillimeter steerable focusing, multifocal, high temporal resolution, coregistration, and the ability to monitor delivered therapy and temperatures in real time. Despite the technical complexity, the future of noninvasive focused ultrasound for neuromodulation as a neuroscience and clinical platform remains bright.
\end{abstract}

Key Words Focused ultrasound $\cdot$ LIFU $\cdot$ HIFU $\cdot$ neuromodulation

\section{Introduction to Ultrasound}

Sound is a mechanical wave of compression and rarefaction of molecules in a medium capable of propagating a wave, such as air, water, or tissue. Humans normally hear sounds in the frequency range between 100 cycles per second $(\mathrm{Hz})$ and $20,000 \mathrm{~Hz}$. Frequencies of sound above the range detectable by the human ear are termed "ultrasound," generally considered those frequencies exceeding $20 \mathrm{kHz}$. Ultrasound typically uses frequencies in the hundreds of thousands or millions of

Invited Review for Innovation and Evolution in Surgical

Neurotherapeutics

Electronic supplementary material The online version of this article (https://doi.org/10.1007/s13311-018-00691-3) contains supplementary material, which is available to authorized users.

David P Darrow

Darro015@umn.edu

1 Department of Neurosurgery, University of Minnesota, 420 Delaware St SE, MMC 96, Room D-429, Minneapolis, MN 55455, USA hertz, but sound can be sustained in some solid materials into the billions of hertz. To generate mechanical oscillations at these frequencies, ultrasound transducers are composed of piezoelectric crystals, which were originally discovered when mechanical pressure applied to the crystals generated electricity. The conversion of mechanical waves into electrical waves, named the piezoelectric effect, was discovered by the Curie brothers in the 19th century, and the suggestion of the inverse piezoelectric effect by Gabriel Lippmann made the field of ultrasound possible [1]. Precise application of an electric current to piezoelectric crystals transduces the energy into precise mechanical movements, generating sound waves.

Acoustic waves at ultrasonic frequencies travel through media in a variety of ways, classified according to the direction that molecules in the media move relative to the direction of propagation of the wave. Longitudinal waves travel along the direction of compression and rarefaction, which is the basis for how sound moves through the air. Longitudinal waves are possible in most materials at any phase. Shear waves travel perpendicularly to the movement of the particles within the medium, much like a string that is rapidly moved back and forth. Each part of the string travels side to side while the wave travels along the string. Shear waves, or transverse 
waves, are sustained primarily within solid materials where the molecular bonds allow forces at right angles but are not seen in simple liquids and gasses.

Ultrasound waves interact variably with the materials they are propagating through, depending on their intrinsic composition. The molecular bonds and structure of materials or tissues determine their resistance to the flow of mechanical waves, termed acoustic impedance. As a result, each material supports a different speed of sound, traveling at approximately $1500 \mathrm{~m} / \mathrm{s}$ in soft biological tissues, $3500 \mathrm{~m} / \mathrm{s}$ in bone, and around $330 \mathrm{~m} / \mathrm{s}$ in air [1]. Mathematically, the acoustic impedance is the product of the speed of sound in the material and the density of the material [2]. As ultrasound passes through the material, the amplitude (or peak pressure) and energy attenuate. Attenuation results from several physical processes that remove energy from ultrasound waves as they propagate through a medium, including scattering, absorption, and mode-conversion. Scattering is caused by inhomogeneities within the medium. Mechanical energy is absorbed by the material through friction to generate heat. Additionally, in some materials and tissues, longitudinal waves are converted into shear waves, or shear waves are converted into longitudinal waves; this process, termed mode-conversion, is particularly salient at the interface of bone and soft tissues, where a significant difference in impedance exists [3].

Because ultrasound propagates as waves, phenomena such as refraction, reflection, interference, and diffraction are produced. As ultrasound encounters changes in materials with different impedances, waves can reflect or refract. The most basic version of diagnostic ultrasound imaging results from delivering short pulses of ultrasound into the body interleaved with periods to capture reflections of the pulses from interfaces between tissues of different impedances. Pulses that are reflected are referred to as echoes, and when echoes are reflected from smooth interfaces between objects of different impedance, the intensity of the echo is proportional to their difference in impedance [2]. The distance traveled can be reconstructed from the time delay of returning echoes of coplanar pulses to reconstruct an image. If the intensity of the echo from the objects in space is displayed as brightness, it is known as a brightness-mode display (B-mode) [2].

Ultrasonic waves also refract when encountering interfaces of media with different impedances and in which the speed of sound is different. This is much like when light refracts across an interface between air and water. Refraction can produce illusions, such as when a straight object passing from air into water appears to bend at the interface. Refraction can produce challenges for medical imaging where some structures appear to be duplicated [2]. Diffraction from inhomogeneities in the tissue scatters ultrasound waves, which can blur reconstructed ultrasound images.

Ultrasound waves can also interfere constructively and destructively with each other. This characteristic fueled the development of diagnostic and therapeutic uses of ultrasound based on control over the interference of ultrasound waves. Arrays of ultrasound transducers spread physically apart can utilize constructive and destructive interference to concentrate energy at a single point [4]. Imaging or therapy using these beams of focused ultrasound is aptly named "focused ultrasound" (FUS). The shape of the focus can be adjusted through small changes in the phase of each of the transducers in the array, called a "phased array." Calculating the phases to generate a desired focus requires an accurate model of the acoustic impedance and the speed of sound in the material and paths that the ultrasound travels. If these paths to the focus include different materials with different speeds of sound, as well as different degrees of attenuation, the waves may produce a wider or more diffuse focus than anticipated. Even if an accurate model is estimated, properties of the material vary with temperature and frequency of the ultrasound wave due to the various forces that shape the dynamic motion of the underlying molecular structure and energy [5]. In certain cases, by interleaving ultrasound imaging with therapeutic ultrasound, termed dual-mode ultrasound, the ultrasound focus can be adjusted in real time to improve the size of the focus [6].

The spatial configuration or geometry of the ultrasound transducers used to form the ultrasound probe can be modified to suit the application. For example, wide, convex arrays allow diverging ultrasound beams to probe larger volumes of tissues and are used in obstetrics to image fetal anatomy, transabdominally [7]. Ultrasound used through burr holes for neurosurgery incorporate closely spaced and unidirectional transducers to image along a straight path through a small window in the skull [8]. Phased arrays became popular for imaging in cardiology because it conferred an improved spatial resolution, which has been particularly useful in capturing images of the actively contracting heart [9]. The advancement of ultrasound technology for the purposes of improving diagnostic imaging of the heart, while in motion, shielded by the chest wall, and carrying blood, provided the necessary foundation for FUS neuromodulation.

In addition to the geometry of the ultrasound array, several other parameters of ultrasound waves can be modulated. As with other sound and electromagnetic waves, the frequency and pressure (amplitude) of ultrasound can be specified or modulated for various applications. The primary frequency, usually the highest, generated is referred to as the carrier frequency, and this can be modulated for various applications. Carrier frequencies for diagnostic ultrasound range from approximately 0.2 to $15 \mathrm{MHz}$. Typically, ultrasound is delivered as short pulses of this carrier frequency to avoid significant thermal energy from being delivered, a method referred to as pulsed ultrasound. The frequency of pulses is the pulse repetition frequency (PRF). Additionally, exposure time refers to the period over which ultrasound pulses are delivered. At high intensities, typical exposure times may be a fraction of a second 
while at lower intensities, exposure times can exceed minutes. Many modern ultrasound platforms also allow multiple carrier frequencies to be simultaneously transmitted to help shape energy delivery by providing further flexibility for steering through certain materials such as bone [10].

\section{Biophysical Effects of Ultrasound}

Forty years after the discovery of piezoelectric crystals, Langevin discovered that a fish could perish when swimming through a beam of high-intensity ultrasound, launching a quest to understand the biophysical effects of ultrasound [1]. Over the past 100 years, ultrasound has gone through many phases of discovery, application, quiescence, and resurgence in the medical field [1]. Despite periods of enthusiastic clinical use and periods of controversy, ultrasound has been established as a foundational platform for medical imaging. To ensure safety while being used for imaging, a significant amount of investigation has focused on understanding the biophysical effects of ultrasound $[1,11]$. The effects of ultrasound on tissue result from the complex biophysical effects of ultrasound with heterogeneous anatomy and physiology still under investigation after many decades of investigation [1]. How ultrasound affects neural tissue is of interest for neuromodulation, but a conclusive biophysical mechanism has not emerged [12]. Biophysical effects of ultrasound in the nervous system have been largely categorized into thermal and nonthermal effects [13-15]. While this categorization is useful for discussion, the effects of ultrasound may simply not be separable in this way. This false dichotomy has confused research on the topic since heat is often generated simultaneously with mechanical effects and vice versa [16].

The generation of thermal energy by ultrasound has been extensively investigated both in vitro and in vivo, and improvements in the mechanistic understanding have been made through computational modeling [17-21]. The temperature induced in tissues by focused ultrasound is dependent on the intensity and the frequency-dependent absorption of the underlying tissue $[1,22]$. Ultrasound offers an inherent tradeoff between penetration depth, resolution, and frequency; higher frequencies have reduced penetration depth but increased spatial resolution $[23,24]$. Tissue response to the thermal energy produced by ultrasound is well characterized with models of impedance and heat transfer, up to temperatures or acoustic intensities that mediate irreversible effects on the proteins in the tissue [18]. Homeostatic cooling, passive perfusion, and diffusion impact tissue heating when introducing thermal energy via ultrasound into perfused tissue [17]. Diffusion can be well modeled with Fick's law, and perfusion has been modeled historically using Pennes' bioheat equation [25]. The heat-generating properties of ultrasound have been applied in the treatment of tissue following musculoskeletal injury, though the therapeutic benefits of this remain controversial [16].

At higher intensities, ultrasound can generate temperatures sufficiently high to denature proteins and coagulate tissues. High-intensity ultrasound has been exploited for clinical applications to ablate kidney stones, fibroids, cancer, or cranial tumors and even to create irreversible functional brain lesions for movement disorders [18, 26-28]. At low intensities, tissue temperature changes are minimal and within the normal physiologic range [29-32]. Numerous studies have reported the absence of any pathological changes from low-intensity focused ultrasound (LIFU), but the effects of small temperature changes in spatially restricted volumes of the nervous system have not been rigorously explored [32]. Even small changes in temperature may have appreciable effects on neural function [27]. The precise definition of pathological temperature rise has not been well characterized and varies by tissue, but guidelines have been published to provide conservative and safe estimates of 1.5 to $2{ }^{\circ} \mathrm{C}$ or an absolute temperature of $41{ }^{\circ} \mathrm{C}[33,34]$. As a result, consistent temperature elevations beyond these safety limits will require application-specific safety studies. It is well established that a rise in temperature increases the rate of enzymatic activity up to the point of denaturation, which begins to occur around $42{ }^{\circ} \mathrm{C}$ [35]. Between 43 and $60{ }^{\circ} \mathrm{C}$, the denaturing of proteins occurs in a logarithmic fashion relative to exposure time [1]. Generally, tissue is damaged at exponentially higher rates as temperature increases in this range and is typically damaged immediately at temperatures above $60^{\circ} \mathrm{C}$. As proteins and cell membranes undergo coagulation at these high temperatures, the acoustic impedance of the tissue also changes in a nonlinear fashion. Direct thermal effects of high-intensity ultrasound lead to changes in tissue that may mediate large and sudden nonthermal effects, which may play a synergistic role in further temperature changes [36].

The nonthermal effects of ultrasound include mechanical pressure, radiation force, and cavitation, as well as organspecific effects such as blood-brain barrier disruption and effects on cell membranes in the nervous system [1, 32, 37]. Nonthermal effects of ultrasound and FUS are less well understood [32]. At high intensities, ultrasound can produce cavitation, where microbubbles grow and collapse with compression and rarefaction. Within biological tissues, cavitation is achieved above a certain threshold, which is dependent on frequency, temperature, and pressure. Cavitation requires nucleation sites where microbubbles can grow, oscillate (stable cavitation), and even sometimes violently collapse (inertial cavitation) [38]. Pre-existing or dissolved gases that are liberated thermally, such as during HIFU, are considered to be the main source of de novo microbubble nucleation sites [38, 39]. Lower intensities of HIFU have also been shown to produce cavitation in vivo, though theoretical calculations for spontaneous cavitation suggest this effect is unlikely during typical 
exposures of diagnostic ultrasound in most tissues [40, 41]. Stable cavitation, when microbubbles oscillate but are not driven to collapse, has been theorized to potentially play a role in affecting cell membrane potentials [14]. Other biophysical effects are seen at intensities sufficient for cavitation and include microstreaming, where acoustic energy drives the movement of liquids in bulk or as more powerful eddy currents [16].

Of special relevance to FUS neuromodulation, the bloodbrain barrier (BBB) is the highly selective interface between the circulatory system and the nervous system where junctions between endothelial cells, astrocytic feet, and pericytes prevent potentially toxic large or hydrophilic molecules from passing into the tightly regulated environment of the central nervous system [42]. Since early reports of the effects of FUS on the brain, it has been known that high-intensity focused ultrasound causes a disruption of the BBB outside of the location of a permanent lesion [43, 44]. Further efforts have demonstrated that FUS can indeed transiently open the BBB to allow delivery of drugs otherwise prevented from reaching the nervous system [45-47]. Through the use of microbubble contrast agents, FUS has been shown to reliably open the BBB at significantly reduced power by using microbubbles for cavitation [46, 47]. The potential of FUS-mediated BBB disruption to mediate numerous types of neuromodulation through the delivery of drugs or genes to a precise focus is vast and provides synergy to traditional and novel biological approaches [48-51].

As a wave, ultrasound can produce a radiation force capable of exerting force on objects or materials [1]. Radiation forces from FUS have been used to assess the composition of unknown materials inside closed containers, providing a method of remote characterization [52]. The effect of acoustic radiation in biological tissues at or near diagnostic imaging thresholds is proportional to the spatial-temporal average of the intensity. Little evidence exists supporting the role of radiation force in vivo, but some authors have postulated that it could play a role in modulating the activity of peripheral nerves $[1,37]$. In contrast, the diagnostic potential of acoustic radiation force has persisted through vibroelastography as well as "acoustical tweezers" for micromanipulation in the laboratory [15]. Ultrasound may also have the potential to directly affect channels and proteins particularly sensitive to mechanical forces, including some types of sodium and potassium channels [53-55].

High-intensity ultrasound is capable of physically pulling apart tissues. Similarly to thermal energy, safety guidelines have been published, though uncertainty remains about precisely defining the safe, mechanical limits before pathological tissue destruction [33, 34]. Estimates of the maximum pressure (mechanical index: MI) and thermal energy (thermal index: TI) exposed to a human constitute the primary safety parameters currently in diagnostic ultrasound, which many use as a guide for LIFU [56].
Effects of Ultrasound on the Nervous System

Designing experiments to characterize the complex biophysical effects of ultrasound at high or low intensities (HIFU/LIFU) on the function of the nervous system poses a particularly difficult challenge [12]. Electrophysiology in vivo during sonication remains challenging due to vibrations of recording electrodes and mechano-electrical coupling that can occur in the tissue [12]. Additionally, flexible and precise ultrasound systems have not been adapted for use in animal models, and those that have been developed require extensive engineering support. Lastly, like electromagnetic waves, the parameter space for ultrasound waves is vast, even when ignoring complex waveforms. Ultrasound transducers produce pulsed or continuous sinusoidal waves, which can produce inherently nonlinear effects in tissues $[18,57,58]$. Novel experimental paradigms must be developed and employed to characterize the effects of ultrasound over this parameter space [59].

There are many challenges to characterize the effects of ultrasound on the nervous system, including nonstationarity, limitations of translation across animal species, and, most obviously, the barrier of the skull. In contrast to the soft and relatively homogenous structure of the brain, the skull has a high acoustic impedance, reflecting a substantial portion of incident ultrasound waves, heating the overlying scalp, and distorting ultrasound through refraction [4]. The concave shape of the skull can naturally lens the remaining acoustic energy. However, the large gradients in acoustic impedance at the interfaces of the skull impede focusing ultrasound directly underneath the skull [21]. Despite these challenges, more recent technical advances in modeling and dual-mode ultrasound have made transcranial ultrasound feasible to control and deliver [6, 21]. In particular, ultrasound transducers can be arranged in one-, two-, or three-dimensional arrays, spaced regularly or randomly, in order to spread the acoustic energy across the skull while precisely timing delivery from each transducer to generate a constructive focus within the brain [60]. Major advancements in computational power have made this process feasible in the past 30 years, and it is now possible to deliver energy by adaptively orchestrating dozens or hundreds of transducers [61]. In particular, dual-mode ultrasound has enabled adaptive closed-loop approaches for correcting distortion from the skull through real-time iterative improvements in the appropriate weighting and timing of each transducer for certain applications [62, 63]. In this way, the individual anatomy of a single patient or animal can be evaluated and accounted for as therapy is delivered in succession, precisely verifying the prescribed dose at the location within predetermined safety margins that may include specific tolerances at sensitive points in the ultrasound field [4]. Lastly, major advancements in three-dimensional stereotaxis have made multimodal investigations using magnetic resonance 
imaging and ultrasound possible through coregistration [64]. Coregistered stereotaxis allows the precise delivery of acoustic energy to loci with higher contrast or highly resolved features from other modalities, such as magnetic resonance imaging (MRI) or computed tomography (CT).

\section{High-Intensity Ultrasound}

Ultrasound delivered at intensities sufficient to damage tissue is termed high-intensity ultrasound, but the particular intensity required to achieve damage varies significantly and is dependent on properties of the tissue and parameters of the acoustical wave [65]. This boundary between high- and low-intensity ultrasound can span significant ultrasound intensities and further work is necessary to apply more sensitive markers of pathology to improve a parameterized understanding of safety and effect margins [33, 34]. Generally, intensities exceeding hundreds of watts per square centimeter applied for a sufficient number of seconds will produce damage, through mechanical destruction, thermal heating, or both [1]. One of the earliest experimental targets of HIFU was the brain, and, even in 1942, well-circumscribed pathological lesions were achieved [66]. As with other methods of introducing thermal energy into the brain, including laser interstitial thermal therapy (LITT), ultrasound delivered at moderate intensities, where tissue temperature is increased to $43-60{ }^{\circ} \mathrm{C}$, produces time-dependent damage to exposed tissue. At higher intensities, tissue is immediately damaged and may demonstrate rapid changes in ultrasonic properties, necessitating real-time adjustments to minimize collateral damage [67]. At high intensities, cavitation may play a significant role in tissue destruction and is often used as a therapeutic endpoint, though this may be an insensitive marker [67, 68]. Cavitation produces inhomogeneities that can substantially affect the ultrasound field. While cavitation can facilitate rapid cell death upon bubble collapse (inertial cavitation), its unpredictable nature necessitates monitoring and/or real-time modeling to be safely controlled [68].

Despite a long history of experimentation with HIFU for lesion formation in the brain, more than 70 years passed before the FDA approved its use for its first indication in the brain. Much of clinically translated HIFU owes acceptance to advances in noninvasive thermometry. Most notably, MR thermometry in combination with advanced modeling allows delivery of prescribed lesion sizes while monitoring safety parameters in real time to facilitate necessary adjustments, termed magnetic resonance-guided focused ultrasound (MRgFUS) [67]. By allowing clinicians the opportunity to monitor the delivery of lesions and specify clinically relevant endpoints and safety margins across anatomical structures, noninvasive delivery of ultrasound has found wider acceptance and interest. Similarly, efforts have been made to employ the use of dual-mode ultrasound to perform noninvasive thermometry and thermography, which carries substantially less cost $[20,69,70]$. Noninvasive temperature imaging, whether based on MR or US, requires basic assumptions about the baseline temperature and employs computational models of relative temperature changes through periodic sampling. By modeling tissue damage through the Arrhenius equation or, similarly, the CEM 43 , thermometry allows precise lesioning of the nervous system [71].

\section{Low-Intensity Ultrasound}

Ultrasound delivered at low intensities has been reported to have a wide range of effects on the nervous system. In vitro studies have demonstrated increased and decreased neuronal firing rate and conduction speeds when FUS is applied [29, $53,72,73]$. In vivo studies have reported ultrasound-evoked responses in animals and humans, though limited, by targeting most of the primary surfaces of the cortex (M1, S1, V1) as well as the hippocampus, thalamus, brainstem, and retina [12, $30,53,74,75]$. Suppression of neural activity by FUS has been described in animals and in humans, most notably on visual and somatosensory evoked potentials, EEG, and seizures [32, 76-81]. Furthermore, others have described variable effects on the peripheral nervous system [12]. Reversible physiologic behavior and lack of pathological changes on histology form the underlying basis for the claim that lowintensity ultrasound provides a safe platform for neuromodulation [32, 37, 65, 82].

Much uncertainty remains about the underlying mechanisms of LIFU neuromodulation [32]. Numerous thermal and nonthermal mechanisms have been proposed. At low intensities, ultrasound creates small changes in temperature that are likely below the capacity of heat transfer from passive perfusion [83]. Consequentially, most studies have attempted to specifically discount the role of thermal effects in both ultrasound-evoked and ultrasound-mediated suppression [12, 30, 84-86]. Few studies have explored the cellular effects of focused thermal energy due to LIFU, but it is plausible that even small changes in temperature modulate excitability given the temperature dependence of membrane potentials of neurons [87]. Additionally, changes in temperature are known to affect ion channel conductance, enzymatic activity, and equilibrium potentials, resulting in complex dynamical changes of neuron firing rates [88-90].

Most authors champion nonthermal mechanical mechanisms of LIFU for neuromodulation. As a mechanical wave, it has been theorized and reported that mechanosensitive ion channels may mediate changes in neuron firing [91, 92]. Some reports have demonstrated changes in voltage-gated calcium, sodium, and potassium channels following LIFU exposures [53-55]. Mechanical wave cavitation has also been proposed to modulate membrane potentials through stable cavitation within the lipid bilayers of neurons [14, 93]. Acoustic radiation pressure has been implicated as a mechanism for 
modulating neural activity with efforts to model the potential dynamics where the effect of the cumulative radiation force suppresses function [31]. Others have comprehensively summarized the increasingly numerous and heterogeneous reports attempting to provide convincing rationale and evidence for an underlying mechanism [12]. While multiple mechanisms almost certainly play a role during LIFU in vivo, the relative contributions and measurable independence of the various mechanisms remains unclear.

Recently, 2 coordinated publications describe the existence of an auditory startle effect, which may account for at least part of the excitatory effects of ultrasound previously reported $[94,95]$. In short, it was found that ultrasound beams generated from single-element transducers with large rostrocaudal extent interacted with the skull base of the animal models during ultrasonication, produced indirect neuromodulation through strong auditory pathway activation [94, 95]. This finding matches well with previous reports identifying that the effect of LIFU is maximized with reduced anesthesia $[30,96,97]$. By introducing powerful mechanical waves into the inner ear, a structure evolved to amplify small mechanical waves, it is concluded that ultrasound delivered in this manner cannot be considered to be mediated by targeted neuromodulation but rather through a startle response. However, the acoustic-startle response does not account for the range of observed neuromodulatory effects of ultrasound, such as those reports in which phased arrays were used (restricted rostrocaudal focus) or in animal models where divergence prevented significant interaction with the skull base [29, $53,74,80,98-100]$. Additionally, it is unclear how the consistently reported finding of suppression of evoked potentials can be accounted for by the acoustic-startle mechanism.

The precise effects of LIFU and the underlying mechanisms continue to remain elusive despite more than 60 years since reversible neuromodulation was initially reported [81]. It is possible that LIFU's primary effect is to suppress neuronal activity, and that excitation by FUS reported previously is entirely due to the acoustic startle responses, though there remains to be a convergence of evidence within the field. In many ways, reliable, spatially restricted suppression could provide an excellent platform for probing or treating the brain and peripheral nervous system. Suppression of active neural circuits implies that FUS could be used to suppress, or functionally disconnect, interconnected regions of the brain and peripheral nervous system, allowing plastic effects to emerge with repeated treatments or longer exposures, especially when paired with behavioral paradigms.

\section{Clinical Utility of FUS for Neuromodulation}

FUS continues to emerge as a versatile platform for neuromodulation. In general, FUS offers many attractive advantages: (1) low cost, (2) noninvasive, (3) steerable, (4) inherent imaging capabilities, (5) compatible and possibly synergistic with other imaging systems, (6) readily adapted for closed-loop therapies, (7) ability to modulate neuronal activity or produce lesions, (8) potential to be used as a platform for targeted drug and gene therapies, and (9) potential for rapid translation to clinical applications as a unified system. Table 1 describes some of the key technologies available for future clinical application. At high intensities, FUS can produce reliable and permanent lesions with results similar to RF ablation. At low intensities, FUS appears to excite or suppress neural circuits in a reversible and noninvasive fashion. While far from being fully characterized, temporarily modulating neural activity at high resolution and low cost would open the door to previously intangible access to the nervous system across a more generalizable and readily available population of human subjects while providing a robust platform for clinical translation for many functional diseases of the human nervous system.

Other platforms for neuromodulation have emerged including transcranial magnetic stimulation (TMS) and transcranial current stimulation (TCS) (see Table 2 for comparison) [59]. While TMS can produce effects localized to around $1-2 \mathrm{~cm}$ at the cortical surface, FUS can achieve submillimeter resolution, limited only by technological advancement. Even higher resolutions may be achieved through harmonic approaches [101]. Transcranial current stimulation also suffers from poor anatomical resolution due to the physical behavior of current and barrier of the skull [102]. The temporal resolution of FUS is similar to or better than these other modalities. Beyond the resolution of an individual focus, there remain only technical restrictions to generate patterned stimuli. Phased arrays can target multiple independent foci, providing a platform for investigation of distributed networks. FUS has already proven itself compatible with other modalities including MRI and EEG and poses significantly fewer challenges than TMS for designing multi-platform experiments or treatments [82]. Dual-mode ultrasound provides an inherent method of real-time imaging for closed-loop monitoring to adjust for patient-specific anatomy and to ensure stable delivery of therapy.

As a result of the plethora of potential advantages of FUS for neuromodulation, reports consistently tout the broad range of possible clinical targets that may be accessible to current and future FUS platforms [21, 32, 37, 59, 65]. These include neurological conditions that are amenable to lesioning, such as brain tumors or movement disorders, as well as those that may require more subtle or network-wide modulation, such as psychiatric disorders. Furthermore, combinations of these effects can be applied as needed; for example, patients with epilepsy may benefit from a combination of HIFU-mediated lesioning 
Table 1 Key terms and technologies for FUS neuromodulation

\begin{tabular}{|c|c|}
\hline HIFU & $\begin{array}{l}\text { High-intensity focused ultrasound creates permanent lesions through coagulation of cellular proteins and thermal ablation that may } \\
\text { include cavitation. Lesion size is monitored through simultaneous monitoring with MR thermometry or US thermometry. } \\
\text { Temperature monitoring provides a method of modeling cell death as therapy is delivered to track the size of the lesion created. } \\
\text { Typical intensities (measured as the peak spatial and average temporal intensity: SPTA) are greater than } 1 \mathrm{~W} / \mathrm{cm}^{2} \text {. }\end{array}$ \\
\hline LIFU & $\begin{array}{l}\text { Low-intensity focused ultrasound modulates the function of the nervous system through the creation of reversible functional lesions } \\
\text { that produce no pathological changes on histological examination. Typical intensities are less than } 1 \mathrm{~W} / \mathrm{cm}^{2} \text {. }\end{array}$ \\
\hline Phased Array & $\begin{array}{l}\text { A set of ultrasound transducers usually in a geometric shape to make use of constructive interference to focus ultrasound at a point. } \\
\text { Small adjustments are made to the phase of each element of the array to adjust for inhomogeneities in materials. }\end{array}$ \\
\hline Dual-Mode & $\begin{array}{l}\text { Dual-mode ultrasound platforms deliver ultrasound at intensities to sufficient to provide therapy and are equipped to interleave periods } \\
\text { of time to also receive reflected ultrasound. Information received can be used to adjust therapy through monitoring of salient features } \\
\text { such as bubble formation or surrogates of temperature change. }\end{array}$ \\
\hline MRgFUS & $\begin{array}{l}\text { Magnetic resonance guided focused ultrasound uses MR thermometry to measure temperature changes induced by ultrasound. While } \\
\text { traditionally used as a method of modeling cell death during the application of HIFU for lesion creation, small temperature changes } \\
\text { of a few degrees could be used to target sites for delivery of LIFU. }\end{array}$ \\
\hline Multifocal FUS & $\begin{array}{l}\text { FUS delivered through phased arrays can steer a focus through the adjustment of the timing of individual elements of the array within a } \\
\text { predefined range based on the number of elements and geometry of the array. Multiple foci can be created simultaneously with only } \\
\text { technical limits on the number of simultaneous reversible or permanent lesions that can be created. Foci can be patterned to } \\
\text { dynamically emerge and decay to investigate network hypotheses. }\end{array}$ \\
\hline $\begin{array}{l}\text { Closed-Loop } \\
\text { FUS }\end{array}$ & $\begin{array}{l}\text { While dual-mode ultrasound may use reflected ultrasound waves to adjust the timing of array elements to improve the size of an } \\
\text { ultrasound focus in a closed loop, physiological data from EEG/fMRI/ECoG could also be incorporated to adjust the intensity or } \\
\text { location of and ultrasound focus. These simultaneous methods of closed-loop operation can simultaneously operate to target specific } \\
\text { loci for various disease states. }\end{array}$ \\
\hline
\end{tabular}

at an epileptogenic focus in addition to LIFU-mediated suppression of other nodes within the network that play a role in the propagation or generation of epileptic activity but have potential adverse side effects when irreversibly lesioned.

\section{Applications of HIFU}

Modern opinions about permanent lesioning for the purpose of changing the function of the nervous system are beginning to recognize benefits such as low-cost and similar efficacy, citing reports from a previous era of functional neurosurgery for movement disorders, pain, and psychiatric disease [103]. If permanent lesions can be safely administered noninvasively, evidence for efficacy may provide a further resurgence of interest in HIFU neuromodulation.
While lesioning is not always considered as a modern form of neuromodulation, some evidence exists that lesioning produces effects equivalent to certain reversible forms of neuromodulation [103]. In particular, unilateral pallidotomy for Parkinson's disease is reported to be equivalent to deep brain stimulation (DBS) while unilateral temporal lobectomy for intractable mesial temporal lobe epilepsy remains the gold standard despite the emergence of the responsive neurostimulation [103, 104]. From a network perspective, lesioning may remove pathologically noisy tissues or sources of pathological activity to improve or restore function. With recent approval and associated technical advancements, HIFU may continue to build momentum for ultrasound as a cornerstone of more widespread neuromodulation as an attractive noninvasive platform.

Table 2 Overview of neuromodulation techniques

\begin{tabular}{llllllll}
\hline & Resolution & Multifocal capability & Temporal resolution & Closed-loop compatible & Invasive & Reversible & Cost \\
\hline LIFU & $\sim 1 \mathrm{~mm}^{*}$ & $\mathrm{Y}$ & $\sim 1 \mathrm{kHz}$ & $\mathrm{Y}$ & $\mathrm{N}$ & $\mathrm{Y}$ & Moderate $^{\dagger}$ \\
TCS & $>500 \mathrm{~mm}$ & $\mathrm{NA}$ & $\sim 1 \mathrm{kHz}$ & $\mathrm{Y}$ & $\mathrm{N}$ & $\mathrm{Y}$ & Low \\
TMS & $10 \mathrm{~mm}$ & Difficult & $\sim 1 \mathrm{~Hz}$ & $\mathrm{~N}$ & $\mathrm{~N}$ & $\mathrm{Y}$ & Moderate \\
DBS & $\sim 1 \mathrm{~mm} *$ & Difficult & $\sim 1 \mathrm{kHz}$ & $\mathrm{Y}$ & $\mathrm{Y}$ & Mostly & High \\
RF ablation & $1-5 \mathrm{~mm}$ & Difficult & $\mathrm{NA}$ & $\mathrm{N}$ & $\mathrm{Y}$ & $\mathrm{N}$ & High \\
HIFU & $\leq 1 \mathrm{~mm}$ & $\mathrm{Y}$ & $\mathrm{NA}$ & $\mathrm{N}$ & $\mathrm{N}$ & Moderate \\
\hline
\end{tabular}

*The theoretical limit of focused ultrasound depends largely on the frequency used and has been shown to be much smaller than $1 \mathrm{~mm}$ with reports on the scale of micrometers while existing clinical systems operate on the $1 \mathrm{~mm}$ scale

${ }^{\dagger}$ The cost of ultrasound equipment remains largely due to the need for creation of specialized arrays and custom control systems that would be susceptible to economies of scale due to materials being readily available 
In many respects, HIFU is optimally suited for treating movement disorders given the known targets in the thalamus and basal ganglia and experience with RF ablation [32]. FUS is delivered most efficiently to deep targets where the skull helps to focus incident acoustic beams. Technical improvements allow millimeter resolution of targets and safe delivery platforms guided by MR thermometry [105]. The FDA has approved MRgFUS for the treatment of patients with essential tremor and is pending approval for Parkinson's disease [106].

While temporal lobectomies remain the gold standard for surgically treating refractory epilepsy, minimally invasive methods of tissue destruction have become increasingly popular through the use of laser interstitial thermal therapy (LITT), in which a fiberoptic catheter is stereotactically placed in the mesial temporal lobe while therapy is monitored using MR thermometry [107]. Patients certainly prefer less invasive approaches, but it is not clear whether LITT is as effective as temporal lobectomy. The application of HIFU for noninvasive temporal lobectomy would be an obvious direction once phased arrays are designed specifically for corridors to the temporal lobe. Because HIFU is noninvasive, stepwise lesion formation could supplant both LITT and temporal lobectomy to provide the appropriate tradeoff between lesion size and adverse effects [108]. The flexibility of progressive noninvasive treatments may allow a super-selective destruction of the mesial temporal lobe, or hippocampus, avoiding significant edema and minimizing total tissue destruction. If the technical challenges of noninvasive HIFU for the temporal lobe are overcome, then noninvasive biomarkers of epilepsy, such as EEG, may become more important as a surrogate for disease states to avoid invasive recordings for seizure localization.

Lesioning for chronic pain syndromes has been extensively pursued with success for specific syndromes including trigeminal neuralgia, as well as injury to the brachial plexus, spinal cord, or peripheral nerves [109, 110]. Lesioning has become increasingly unpopular and the decreased use of surgical treatments for pain has been a byproduct, without emerging alternative therapies [103]. Not only would HIFU increase accessibility to lesioning for pain, but novel discoveries from LIFU mapping could facilitate personalized approaches towards comprehensive pain treatment. Already, studies are emerging to tackle some pain syndromes with ultrasound [111]. Despite the challenge of delivering FUS through the complex bony anatomy of the spine, work has begun to establish the approach [112].

\section{Applications for LIFU}

Low-intensity FUS is less developed and understood than lesioning with HIFU, but it has the potential to supplant many current therapies and lead clinical translation for a much wider range of disorders of the nervous system due to its reversibility and safety [12]. As a noninvasive and reversible method of neuromodulation with submillimeter resolution across the brain, anatomical approaches towards investigating the basis of neural function could become widely accessible across neuroscience and cognitive research. LIFU can be coregistered with anatomy through dual-mode systems or with stereotactic systems. Additionally, LIFU has already been demonstrated to be compatible with MR and EEG systems and can be used to complement these technologies, allowing focal stimulation and fast temporal acquisition. Future systems will allow multifocal therapy, facilitating novel approaches towards investigating network models of human disease. Effects of LIFU on the autonomic and peripheral nervous systems allow a holistic platform for discovery of the underlying basis for diseases of the nervous system and translation into clinical treatments.

The research paradigm that LIFU may provide is complemented by its ease of translation into clinical and personalizable treatments. As a safe research platform, LIFU may also establish itself as a relatively safe clinical platform that will evolve into portable versions as implantable and wearable ultrasound arrays [113, 114]. While initially LIFU may be offered in a fashion similar to TMS with numerous, relatively low-cost devices available at clinical centers, ultrasound does not suffer from the limitations of the necessary hardware to generate a high magnetic field, and clever technical innovations may facilitate lower energy requirements [12].

Importantly, as an indirect but potentially powerful method of neuromodulation, the role of FUS in BBB opening and the ability to deliver focal drugs or genes may even supplant the direct neuromodulatory effects of FUS. The BBB is a common obstacle in the development of neuroactive pharmacological compounds, and ultrasound has the potential to change the types or dosages of medications needed to produce clinically beneficial effects while minimizing side effects by allowing for temporary or intermittent disruption of the BBB.

While a few early studies have reported meaningful progress towards the use of ultrasound for disease models of pain and epilepsy, the application of ultrasonic neuromodulation to treat other diseases of the nervous system, including movement disorders, psychiatric disorders, and even disorders indirectly regulated by neural mechanisms, such as obesity or hypertension, remains hopeful but speculative $[77,111]$.

The pursuit of LIFU as a robust platform for neuromodulation will require key technical improvements in combination with further mechanistic clarity. Phased arrays of ultrasound transducers provide a significant advantage over single-element transducers through resolution and energy density at the scalp. One- and two-dimensional arrays are currently in use and consist of regularly spaced transducers over the surface of an arch or hemisphere [115]. These rigid arrays are not specific to the shape of the skull or maximally dense [115]. Significant improvements could be achieved through platforms capable of incorporating dense and quasirandom arrays, 
customizable to complex anatomy where specific anatomical variations can be utilized maximally [115]. Major advances have already been reported in array technology $[60,116]$. Technological advances to make transducers smaller, more efficient, and more precise would add to the feasibility of clinical LIFU.

The use of large numbers of transducers at high density offers more flexibility than single probes, but it also adds additional computational complexity, especially when used in a closed loop for adaptive focusing. Platforms and efficiently parallelizable algorithms capable of supporting thousands of independently controlled transducers will be needed in order to implement high-density FUS arrays, which has been reported [117]. Modeling may also become increasingly important as nonlinear ultrasound effects are exploited or suppressed for various applications. Especially with the current trend towards super-resolution ultrasound beams that are capable of detecting features as small as a micron, modeling will be necessary to efficiently reconstruct data and improve the signal to noise ratio $[118,119]$.

\section{Conclusions}

In so many ways, FUS neuromodulation is remarkable. By sculpting high-frequency sound, it is now possible to precisely destroy or dynamically and reversibly modulate the activity of 1 or many millimeter voxel(s) deep within the brain without ever touching the skull or making an incision. Ethical surgeons dream of treating or curing a patient while removing the risk and suffering of neurosurgery. While the precise mechanism of LIFU neuromodulation remains elusive, as the evidence for safety and neuromodulation build, the clinical utility of FUS neuromodulation will be revealed by physicians across the disciplines of neurosurgery, psychiatry, neurology, and anesthesia as clinically accessible platforms become available. Careful studies with real-time monitoring of thermal and mechanical doses can progressively establish the safety of LIFU in humans to encourage disease-specific application while pioneers of LIFU neuromodulation can continue to tease apart the interdependent mechanisms. Complimentary potential exists for the use of FUS neuromodulation across the range of basic and cognitive neurosciences for discovery and translation.

Readily accessible clinical targets for FUS neuromodulation include diseases with better known anatomical targets such as movement disorders, epilepsy, and pain, and FUS neuromodulation is poised to more comprehensively investigate and advance the treatment of psychiatric diseases. Phased arrays, dual-mode operation, and stereotaxis through coregistration are emerging as a nexus of technologies promising safety and flexibility for network-informed investigations and treatments of these more distributed diseases. The ease of incorporating biomarker mapping and real-time feedback may complement or surpass efforts to do the same for existing clinical modalities such as DBS [120-122]. FUS may even play a key role in providing targeted and transient blood-brain barrier interruption to deliver tailored drug and gene therapies for neurodegenerative and neoplastic processes.

Overall, FUS is extremely promising as a cost-effective, noninvasive, precise, translatable, and flexible platform for neuromodulation. With improved mechanistic understanding and continued technological improvements, FUS has the potential to advance our understanding of nervous system function and change the standard of care for a wide variety of neurological and psychiatric conditions.

Required Author Forms Disclosure forms provided by the authors are available with the online version of this article.

\section{References}

1. O'Brien WD Jr. Ultrasound-biophysics mechanisms. Prog. Biophys. Mol. Biol. 2007;93:212-255.

2. Chan V, Perlas A. Basics of Ultrasound Imaging. In: Narouze SN, editor. Atlas of Ultrasound-Guided Procedures in Interventional Pain Management. New York, NY: Springer New York; 2011. p. 13-19.

3. Pinton G, Aubry J-F, Bossy E, et al. Attenuation, scattering, and absorption of ultrasound in the skull bone. Med. Phys. 2012;39: 299-307.

4. Harary M, Segar DJ, Huang KT, et al. Focused ultrasound in neurosurgery: a historical perspective. Neurosurg. Focus. 2018;44:E2.

5. Duck FA. Nonlinear acoustics in diagnostic ultrasound. Ultrasound Med. Biol. 2002;28:1-18.

6. Liu D, Casper A, Haritonova A, et al. Adaptive lesion formation using dual mode ultrasound array system. AIP Conf. Proc. 2017;1821:060003.

7. Newman PG, Rozycki GS. The history of ultrasound. Surg. Clin. North Am. 1998;78:179-195.

8. Strowitzki M, Moringlane JR, Steudel W. Ultrasound-based navigation during intracranial burr hole procedures: experience in a series of 100 cases. Surg. Neurol. 2000;54:134-144.

9. Meyer RA. History of ultrasound in cardiology. J. Ultrasound Med. 2004;23:1-11.

10. Sun XL, Yan JP, Li YF, et al. Multi-frequency ultrasound transducers for medical applications: a survey. International Journal of Intelligent Robotics and Applications [Internet]. 2018. https://doi. org/10.1007/s41315-018-0057-7.

11. Shankar H, Pagel PS. Potential Adverse Ultrasound-related Biological EffectsA Critical Review. Anesthesiology. 2011;115: 1109-1124.

12. Naor O, Krupa S, Shoham S. Ultrasonic neuromodulation. J. Neural Eng. 2016;13:031003.

13. Dalecki D. Mechanical bioeffects of ultrasound. Annu. Rev. Biomed. Eng. 2004;6:229-248.

14. Krasovitski B, Frenkel V, Shoham S, et al. Intramembrane cavitation as a unifying mechanism for ultrasound-induced bioeffects. Proc. Natl. Acad. Sci. U. S. A. 2011;108:3258-3263.

15. Sarvazyan AP, Rudenko OV, Nyborg WL. Biomedical applications of radiation force of ultrasound: historical roots and physical basis. Ultrasound Med. Biol. 2010;36:1379-1394. 
16. Baker KG, Robertson VJ, Duck FA. A review of therapeutic ultrasound: biophysical effects. Phys. Ther. 2001;81:1351-1358.

17. Rossmanna C, Haemmerich D. Review of temperature dependence of thermal properties, dielectric properties, and perfusion of biological tissues at hyperthermic and ablation temperatures. Crit. Rev. Biomed. Eng. 2014;42:467-492.

18. Wojcik G, Mould J, Abboud N, et al. Nonlinear modeling of therapeutic ultrasound. 1995 IEEE Ultrasonics Symposium. Proceedings. An International Symposium. ieeexplore.ieee.org; 1995. p. 1617-1622 vol.2.

19. Pinton G, Pernot M, Bossy E, et al. Mechanisms of attenuation and heating dissipation of ultrasound in the skull bone: Comparison between simulation models and experiments. 2010 IEEE International Ultrasonics Symposium. 2010. p. 225-228.

20. Seip R, Ebbini ES. Noninvasive estimation of tissue temperature response to heating fields using diagnostic ultrasound. IEEE Trans. Biomed. Eng. 1995;42:828-839.

21. Krishna V, Sammartino F, Rezai A. A Review of the Current Therapies, Challenges, and Future Directions of Transcranial Focused Ultrasound Technology: Advances in Diagnosis and Treatment. JAMA Neurol. 2018;75:246-254.

22. Smith NB, Webb AG, Ellis DS, et al. Experimental verification of theoretical in vivo ultrasound heating using cobalt detected magnetic resonance. IEEE Trans. Ultrason. Ferroelectr. Freq. Control. 1995;42:489-491.

23. Draper DO, Castel JC, Castel D. Rate of temperature increase in human muscle during $1 \mathrm{MHz}$ and $3 \mathrm{MHz}$ continuous ultrasound. J. Orthop. Sports Phys. Ther. 1995;22:142-150.

24. Ng A, Swanevelder J. Resolution in ultrasound imaging. Contin Educ Anaesth Crit Care Pain. 2011;11:186-192.

25. Pennes HH. Analysis of tissue and arterial blood temperatures in the resting human forearm. J. Appl. Physiol. 1948;1:93-122.

26. Evans KD, Weiss B, Knopp M. High-Intensity Focused Ultrasound (HIFU) for Specific Therapeutic Treatments: A Literature Review. J. Diagn. Med. Sonogr. 2007;23:319-327.

27. Elias WJ, Huss D, Voss T, et al. A pilot study of focused ultrasound thalamotomy for essential tremor. N. Engl. J. Med. 2013;369:640-648.

28. Ikeda T, Yoshizawa S, Koizumi N, et al. Focused Ultrasound and Lithotripsy. Adv. Exp. Med. Biol. 2016;880:113-129.

29. Rinaldi PC, Jones JP, Reines F, et al. Modification by focused ultrasound pulses of electrically evoked responses from an in vitro hippocampal preparation. Brain Res. 1991;558:36-42.

30. Tufail Y, Matyushov A, Baldwin N, et al. Transcranial pulsed ultrasound stimulates intact brain circuits. Neuron. 2010;66:681694.

31. Wahab RA, Choi M, Liu Y, et al. Mechanical bioeffects of pulsed high intensity focused ultrasound on a simple neural model: Bioeffects of pulsed ultrasound on nerves. Med. Phys. 2012;39: 4274-4283.

32. Baek H, Pahk KJ, Kim H. A review of low-intensity focused ultrasound for neuromodulation. Biomed. Eng. Lett. 2017;7: 135-142.

33. Barnett SB, Ter Haar GR, Ziskin MC, et al. International recommendations and guidelines for the safe use of diagnostic ultrasound in medicine. Ultrasound Med. Biol. 2000;26:355-366.

34. Nelson TR, Fowlkes JB, Abramowicz JS, et al. Ultrasound biosafety considerations for the practicing sonographer and sonologist. J. Ultrasound Med. 2009;28:139-150.

35. Dickson JA, Calderwood SK. Temperature range and selective sensitivity of tumors to hyperthermia: a critical review. Ann. N. Y. Acad. Sci. 1980;335:180-205.

36. Kyriakou Z, Corral-Baques MI, Amat A, et al. HIFU-induced cavitation and heating in ex vivo porcine subcutaneous fat. Ultrasound Med. Biol. 2011;37:568-579.
37. Tyler WJ, Lani SW, Hwang GM. Ultrasonic modulation of neural circuit activity. Curr. Opin. Neurobiol. 2018;50:222-231.

38. Neppiras EA. Acoustic cavitation series: part one: Acoustic cavitation: an introduction. Ultrasonics. 1984;22:25-28.

39. Izadifar Z, Babyn P, Chapman D. Mechanical and Biological Effects of Ultrasound: A Review of Present Knowledge. Ultrasound in Medicine and Biology. 2017;43:1085-1104.

40. Church CC. Spontaneous homogeneous nucleation, inertial cavitation and the safety of diagnostic ultrasound. Ultrasound Med. Biol. 2002;28:1349-1364.

41. Holland CK, Deng CX, Apfel RE, et al. Direct evidence of cavitation in vivo from diagnostic ultrasound. Ultrasound Med. Biol. 1996;22:917-925.

42. Abbott NJ, Rönnbäck L, Hansson E. Astrocyte-endothelial interactions at the blood-brain barrier. Nat. Rev. Neurosci. 2006;7:41.

43. Bakay L, Ballantine HT Jr, Hueter TF, et al. Ultrasonically produced changes in the blood-brain barrier. AMA Arch. Neurol. Psychiatry. 1956;76:457-467.

44. Patrick JT, Nolting MN, Goss SA, et al. Ultrasound and the bloodbrain barrier. Adv. Exp. Med. Biol. 1990;267:369-381.

45. Ballantine HT Jr, Bell E, Manlapaz J. Progress and problems in the neurological applications of focused ultrasound. J. Neurosurg. 1960;17:858-876.

46. Hynynen K, McDannold N, Vykhodtseva N, et al. Noninvasive MR imaging-guided focal opening of the blood-brain barrier in rabbits. Radiology. 2001;220:640-646.

47. Yang F-Y, Lin Y-S, Kang K-H, et al. Reversible blood-brain barrier disruption by repeated transcranial focused ultrasound allows enhanced extravasation. J. Control. Release. 2011;150:111-116.

48. Airan RD, Meyer RA, Ellens NPK, et al. Noninvasive Targeted Transcranial Neuromodulation via Focused Ultrasound Gated Drug Release from Nanoemulsions. Nano Lett. 2017;17:652-659.

49. Downs ME, Buch A, Karakatsani ME, et al. Blood-Brain Barrier Opening in Behaving Non-Human Primates via Focused Ultrasound with Systemically Administered Microbubbles. Sci. Rep. 2015;5:15076.

50. Wang S, Kugelman T, Buch A, et al. Non-invasive, Focused Ultrasound-Facilitated Gene Delivery for Optogenetics, Sci. Rep. 2017;7:39955.

51. Mead B, Kim N, Negron K, et al. Intersections of neuromodulation, focused ultrasound, and gene delivery with brain-penetrating nanoparticles. J. Acoust. Soc. Am. 2017;142: 2669-2669.

52. Rudenko OV, Sarvazyan AP, Emelianov SY. Acoustic radiation force and streaming induced by focused nonlinear ultrasound in a dissipative medium. J. Acoust. Soc. Am. 1996;99:2791-2798.

53. Tyler WJ, Tufail Y, Finsterwald M, et al. Remote excitation of neuronal circuits using low-intensity, low-frequency ultrasound. PLoS One. 2008;3:e3511.

54. Kubanek J, Shi J, Marsh J, et al. Ultrasound modulates ion channel currents. Sci. Rep. 2016;6:24170.

55. Kubanek J, Shukla P, Das A, et al. Ultrasound Elicits Behavioral Responses through Mechanical Effects on Neurons and Ion Channels in a Simple Nervous System. J. Neurosci. 2018;38: 3081-3091.

56. Duck FA. The Meaning of Thermal Index (TI) and Mechanical Index (MI) Values. BMUS Bulletin. 1997;5:36-40.

57. Humphrey VF. Nonlinear propagation in ultrasonic fields: measurements, modelling and harmonic imaging. Ultrasonics. 2000;38:267-272.

58. Zemp RJ, Tavakkoli J, Cobbold RSC. Modeling of nonlinear ultrasound propagation in tissue from array transducers. J. Acoust. Soc. Am. 2003;113:139-152.

59. Kubanek J. Neuromodulation with transcranial focused ultrasound. Neurosurg. Focus. 2018;44:E14. 
60. Hand JW, Shaw A, Sadhoo N, et al. A random phased array device for delivery of high intensity focused ultrasound. Phys. Med. Biol. 2009;54:5675-5693.

61. Techavipoo U, Worasawate D, Boonleelakul W, et al. Toward Optimal Computation of Ultrasound Image Reconstruction Using CPU and GPU. Sensors [Internet]. 2016;16. Available from: https://doi.org/10.3390/s16121986.

62. Ebbini ES, Yao H, Shrestha A. Dual-mode ultrasound phased arrays for image-guided surgery. Ultrason. Imaging. 2006;28: $65-82$.

63. Mueller JK, Ai L, Bansal P, et al. Numerical evaluation of the skull for human neuromodulation with transcranial focused ultrasound. J. Neural Eng. 2017;14:066012.

64. Magnin R, Rabusseau F, Salabartan F, et al. Magnetic resonanceguided motorized transcranial ultrasound system for blood-brain barrier permeabilization along arbitrary trajectories in rodents. $\mathrm{J}$ Ther Ultrasound. 2015;3:22.

65. Bystritsky A, Korb AS, Douglas PK, et al. A review of lowintensity focused ultrasound pulsation. Brain Stimul. 2011;4: 125-136.

66. Lynn JG, Zwemer RL, Chick AJ, et al. A new method for the generation and use of focused ultrasound in experimental biology. J. Gen. Physiol. 1942;26:179-193.

67. Ye G, Smith PP, Noble JA. Model-based ultrasound temperature visualization during and following HIFU exposure. Ultrasound Med. Biol. 2010;36:234-249.

68. Gyöngy M, Coussios C-C. Passive spatial mapping of inertial cavitation during HIFU exposure. IEEE Trans. Biomed. Eng. 2010;57:48-56.

69. Miller NR, Bamber JC, ter Haar GR. Imaging of temperatureinduced echo strain: preliminary in vitro study to assess feasibility for guiding focused ultrasound surgery. Ultrasound Med. Biol. 2004;30:345-356.

70. Simon C, Vanbaren P, Ebbini ES. Two-dimensional temperature estimation using diagnostic ultrasound. IEEE Trans. Ultrason. Ferroelectr. Freq. Control. 1998;45:1088-1099.

71. Yung JP, Shetty A, Elliott A, et al. Quantitative comparison of thermal dose models in normal canine brain. Med. Phys. 2010;37:5313-5321.

72. Takagi SF, Higashino S, Shibuya T, et al. The actions of ultrasound on the myelinated nerve, the spinal cord and the brain. Jpn. J. Physiol. 1960;10:183-193.

73. Tsui P-H, Wang S-H, Huang C-C. In vitro effects of ultrasound with different energies on the conduction properties of neural tissue. Ultrasonics. 2005;43:560-565.

74. Legon W, Sato TF, Opitz A, et al. Transcranial focused ultrasound modulates the activity of primary somatosensory cortex in humans. Nat. Neurosci. 2014;17:322-329.

75. Gulick DW, Li T, Kleim JA, et al. Comparison of Electrical and Ultrasound Neurostimulation in Rat Motor Cortex. Ultrasound Med. Biol. 2017;43:2824-2833.

76. Daniels D, Sharabi S, Last D, et al. Focused Ultrasound-Induced Suppression of Auditory Evoked Potentials in Vivo. Ultrasound Med. Biol. 2018;44:1022-1030.

77. Min B-K, Bystritsky A, Jung K-I, et al. Focused ultrasoundmediated suppression of chemically-induced acute epileptic EEG activity. BMC Neurosci. 2011;12:23.

78. Chu P-C, Liu H-L, Lai H-Y, et al. Neuromodulation accompanying focused ultrasound-induced blood-brain barrier opening. Sci. Rep. 2015;5:15477.

79. Kim H, Park MY, Lee SD, et al. Suppression of EEG visualevoked potentials in rats through neuromodulatory focused ultrasound. Neuroreport. 2015;26:211-215.

80. Dallapiazza RF, Timbie KF, Holmberg S, et al. Noninvasive neuromodulation and thalamic mapping with low-intensity focused ultrasound. J. Neurosurg. 2017;1-10.
81. Fry FJ, Ades HW, Fry WJ. Production of reversible changes in the central nervous system by ultrasound. Science. 1958;127:83-84.

82. Rezayat E, Toostani IG. A Review on Brain Stimulation Using Low Intensity Focused Ultrasound. Basic Clin Neurosci. 2016;7: 187-194.

83. Dinno MA, Dyson M, Young SR, et al. The significance of membrane changes in the safe and effective use of therapeutic and diagnostic ultrasound. Phys. Med. Biol. 1989;34:1543-1552.

84. Yoo S-S, Bystritsky A, Lee J-H, et al. Focused ultrasound modulates region-specific brain activity. Neuroimage. 2011;56:12671275.

85. Kim H, Taghados SJ, Fischer K, et al. Noninvasive transcranial stimulation of rat abducens nerve by focused ultrasound. Ultrasound Med. Biol. 2012;38:1568-1575.

86. Wright CJ, Rothwell J, Saffari N. Ultrasonic stimulation of peripheral nervous tissue: an investigation into mechanisms. J. Phys. Conf. Ser. 2015;581:012003.

87. Buzatu S. The temperature-induced changes in membrane potential. Riv. Biol. 2009;102:199-217.

88. Borrelli MJ, Bailey KI, Dunn F. Early ultrasonic effects upon mammalian CNS structures (chemical synapses). J. Acoust. Soc. Am. 1981;69:1514-1516.

89. Juan EJ, González R, Albors G, et al. Vagus Nerve Modulation Using Focused Pulsed Ultrasound: Potential Applications and Preliminary Observations in a Rat. Int. J. Imaging Syst. Technol. 2014;24:67-71.

90. Lele PP. Effects of focused ultrasonic radiation on peripheral nerve, with observations on local heating. Exp. Neurol. 1963;8: 47-83.

91. Tyler WJ. The mechanobiology of brain function. Nat. Rev. Neurosci. 2012;13:867-878.

92. Ye J, Tang S, Meng L, et al. Ultrasonic Control of Neural Activity through Activation of the Mechanosensitive Channel MscL. Nano Lett. 2018;18:4148-4155.

93. Plaksin M, Shoham S, Kimmel E. Intramembrane Cavitation as a Predictive Bio-Piezoelectric Mechanism for Ultrasonic Brain Stimulation. Phys. Rev. X. 2014;4:011004.

94. Sato T, Shapiro MG, Tsao DY. Ultrasonic Neuromodulation Causes Widespread Cortical Activation via an Indirect Auditory Mechanism. Neuron. 2018;98:1031-1041.e5.

95. Guo H, Hamilton M 2nd, Offutt SJ, et al. Ultrasound Produces Extensive Brain Activation via a Cochlear Pathway. Neuron. 2018;98:1020-1030.e4.

96. Mehić E, Xu JM, Caler CJ, et al. Increased Anatomical Specificity of Neuromodulation via Modulated Focused Ultrasound. PLoS One. 2014;9:e86939.

97. Tufail Y, Yoshihiro A, Pati S, et al. Ultrasonic neuromodulation by brain stimulation with transcranial ultrasound. Nat. Protoc. 2011;6:1453-1470.

98. Khraiche ML, Phillips WB, Jackson N, et al. Ultrasound induced increase in excitability of single neurons. Conf. Proc. IEEE Eng. Med. Biol. Soc. 2008;2008:4246-4249.

99. Legon W, Bansal P, Tyshynsky R, et al. Transcranial focused ultrasound neuromodulation of the human primary motor cortex. Sci. Rep. 2018;8:10007.

100. Legon W, Ai L, Bansal P, et al. Neuromodulation with singleelement transcranial focused ultrasound in human thalamus. Hum. Brain Mapp. 2018;39:1995-2006.

101. Filonenko EA, Khokhlova VA. Effect of acoustic nonlinearity on heating of biological tissue by high-intensity focused ultrasound. Acoust. Phys. 2001;47:468-475.

102. Miranda PC. Physics of effects of transcranial brain stimulation. Handb. Clin. Neurol. 2013;116:353-366.

103. Hariz MI, Hariz G-M. Therapeutic stimulation versus ablation. Handb. Clin. Neurol. 2013;116:63-71. 
104. Van Ness P, Skarpaas TC, Morrell M. Long-Term Outcome of Adults with Medically Intractable Mesial Temporal Lobe Seizures Treated with Responsive Neurostimulation (S52.001). Neurology. 2016;86:S52.001.

105. Khanna N, Gandhi D, Steven A, et al. Intracranial Applications of MR Imaging-Guided Focused Ultrasound. AJNR Am. J. Neuroradiol. [Internet]. 2016 [cited 2018 Jul 15]; Available from: http://www.ajnr.org/content/early/2016/08/18/ajnr.A4902. abstract.

106. Fishman PS. Thalamotomy for essential tremor: FDA approval brings brain treatment with FUS to the clinic. J Ther Ultrasound. 2017;5:19.

107. Shukla ND, Ho AL, Pendharkar AV, et al. Laser interstitial thermal therapy for the treatment of epilepsy: evidence to date. Neuropsychiatr. Dis. Treat. 2017;13:2469-2475.

108. Schramm J. Temporal lobe epilepsy surgery and the quest for optimal extent of resection: a review. Epilepsia. 2008;49:12961307.

109. Rath SA, Braun V, Soliman N, et al. Results of DREZ coagulations for pain related to plexus lesions, spinal cord injuries and postherpetic neuralgia. Acta Neurochir. . 1996;138:364-369.

110. Mullan S, Lichtor T. Percutaneous microcompression of the trigeminal ganglion for trigeminal neuralgia. J. Neurosurg. 1983;59: 1007-1012.

111. Monteith SJ, Medel R, Kassell NF, et al. Transcranial magnetic resonance-guided focused ultrasound surgery for trigeminal neuralgia: a cadaveric and laboratory feasibility study. J. Neurosurg. 2013;118:319-328.

112. Payne AH, Hawryluk GW, Anzai Y, et al. Magnetic resonance imaging-guided focused ultrasound to increase localized bloodspinal cord barrier permeability. Neural Regeneration Res. 2017;12:2045-2049.

113. Horodyckid C, Canney M, Vignot A, et al. Safe long-term repeated disruption of the blood-brain barrier using an implantable ultrasound device: a multiparametric study in a primate model. J. Neurosurg. 2017;126:1351-1361.

114. Hwang GM, Lani SW, Rosenberg AP, et al. Forward-looking engineering concepts for ultrasonic modulation of neural circuit activity in humans. Micro- and Nanotechnology Sensors, Systems, and Applications X. International Society for Optics and Photonics; 2018. p. 106391J.

115. Hynynen K, Jones RM. Image-guided ultrasound phased arrays are a disruptive technology for non-invasive therapy. Phys. Med. Biol. 2016;61:R206-R248.

116. Rosnitskiy PB, Vysokanov BA, Gavrilov LR, et al. Method for Designing Multielement Fully Populated Random Phased Arrays for Ultrasound Surgery Applications. IEEE Trans. Ultrason. Ferroelectr. Freq. Control. 2018;65:630-637.

117. Hynynen K, Clement GT, McDannold N, et al. 500-element ultrasound phased array system for noninvasive focal surgery of the brain: a preliminary rabbit study with ex vivo human skulls. Magn. Reson. Med. 2004;52:100-107.

118. Viessmann OM, Eckersley RJ, Christensen-Jeffries K, et al. Acoustic super-resolution with ultrasound and microbubbles. Phys. Med. Biol. 2013;58:6447-6458.

119. Errico C, Pierre J, Pezet S, et al. Ultrafast ultrasound localization microscopy for deep super-resolution vascular imaging. Nature. 2015;527:499-502.

120. Hamani C, Richter E, Schwalb JM, et al. Bilateral subthalamic nucleus stimulation for Parkinson's disease: a systematic review of the clinical literature. Neurosurgery. 2005;56:1313-1321; discussion 1321-1324.

121. Rosin B, Slovik M, Mitelman R, et al. Closed-loop deep brain stimulation is superior in ameliorating parkinsonism. Neuron. 2011;72:370-384.

122. Little S, Pogosyan A, Neal S, et al. Adaptive deep brain stimulation in advanced Parkinson disease. Ann. Neurol. 2013;74:449457. 